\title{
Aldo-keto reductase (AKR) superfamily: Genomics and annotation
}

\author{
Rebekka D. Mindnich and Trevor M. Penning* \\ Center for Excellence in Environmental Toxicology, Department of Pharmacology, University of Pennsylvania, School of Medicine, \\ Philadelphia, PA 19104-6084, USA \\ *Correspondence to: Tel: +1 215898 9445; Fax: +1 215573 2236; E-mail: penning@mail.med.upenn.edu
}

Date received (in revised form): 22nd May 2009

\begin{abstract}
Aldo-keto reductases (AKRs) are phase I metabolising enzymes that catalyse the reduced nicotinamide adenine dinucleotide (phosphate) (NAD $(\mathrm{P}) \mathrm{H})$-dependent reduction of carbonyl groups to yield primary and secondary alcohols on a wide range of substrates, including aliphatic and aromatic aldehydes and ketones, ketoprostaglandins, ketosteroids and xenobiotics. In so doing they functionalise the carbonyl group for conjugation (phase II enzyme reactions). Although functionally diverse, AKRs form a protein superfamily based on their high sequence identity and common protein fold, the $(\alpha / \beta)_{8}$-barrel structure. Well over I50 AKR enzymes, from diverse organisms, have been annotated so far and given systematic names according to a nomenclature that is based on multiple protein sequence alignment and degree of identity. Annotation of non-vertebrate AKRs at the National Center for Biotechnology Information or Vertebrate Genome Annotation (vega) database does not often include the systematic nomenclature name, so the most comprehensive overview of all annotated AKRs is found on the AKR website (http://www.med.upenn.edu/akr/). This site also hosts links to more detailed and specialised information (eg on crystal structures, gene expression and single nucleotide polymorphisms [SNPs]). The proteinbased AKR nomenclature allows unambiguous identification of a given enzyme but does not reflect the wealth of genomic and transcriptomic variation that exists in the various databases. In this context, identification of putative new AKRs and their distinction from pseudogenes are challenging. This review provides a short summary of the characteristic features of AKR biochemistry and structure that have been reviewed in great detail elsewhere, and focuses mainly on nomenclature and database entries of human AKRs that so far have not been subject to systematic annotation. Recent developments in the annotation of SNP and transcript variance in AKRs are also summarised.
\end{abstract}

Keywords: carbonyl reduction, nomenclature, pseudogene, SNP, splice variant

\section{Introduction}

Aldo-keto reductases (AKRs) form a superfamily of proteins characterised by their common threedimensional structure and reaction mechanism in catalysing the reduced nicotinamide adenine dinucleotide (phosphate) (NAD(P)H)-dependent oxido-reduction of carbonyl groups. ${ }^{1,2}$ More than 150 enzymes have been identified in a wide range of organisms, including prokaryotes and eukaryotes such as plants, fungi and vertebrates. Based on sequence identity, the proteins fall into 15 different families, termed AKR1-AKR15, and some families (eg AKR1) contain multiple subfamilies. ${ }^{1,3,4}$ Fourteen human AKRs (Table 1), which fall into families 1, 6 and 7, have been annotated and their specific structural and kinetic features, as well as substrate and genomic characteristics, have been recently and comprehensively reviewed. ${ }^{5,6}$

AKRs are, for the most part, cytosolic and are active as monomers. So far, only AKR2, AKR6 
Table I. Systematic and trivial names of human Aldo-keto reductases (AKRs)

\begin{tabular}{|c|c|}
\hline $\begin{array}{l}\text { Systematic } \\
\text { name }\end{array}$ & $\begin{array}{l}\text { Trivial name/often-used } \\
\text { abbreviations }\end{array}$ \\
\hline AKRIAI & Aldehyde reductase; ALR; ALDR I \\
\hline$A K R I B I$ & Aldose reductase; AR, ALR2 \\
\hline AKRIBIO & $\begin{array}{l}\text { Small intestine-like aldose reductase; } \\
\text { ARL-I }\end{array}$ \\
\hline $\mathrm{AKRICI}$ & $\begin{array}{l}\text { Dihydrodiol dehydrogenase I; } \\
20 \alpha,(3 \alpha) \text {-Hydroxysteroid dehydrogenase }\end{array}$ \\
\hline AKRIC2 & $\begin{array}{l}\text { Dihydrodiol dehydrogenase } 2 \text {; } \\
3 \alpha \text {-Hydroxysteroid dehydrogenase, } \\
\text { type III }\end{array}$ \\
\hline AKRIC3 & $3 \alpha-$ Hydroxysteroid dehydrogenase, type II \\
\hline AKRIC4 & $\begin{array}{l}\text { Dihydrodiol dehydrogenase } 4 ; \\
\text { chlordecone reductase; } \\
3 \alpha \text {-hydroxysteroid dehydrogenase, type I }\end{array}$ \\
\hline AKRIDI & $\Delta^{4}$-3-Ketosteroid-5 $\beta$-reductase \\
\hline AKRIE2 & $\begin{array}{l}\text { Aldo-keto reductase family I, member } \\
\text { C-like 2; AKRICL2 }\end{array}$ \\
\hline AKR6A3 & $\begin{array}{l}\text { KCNAB I; hKvbeta3; potassium } \\
\text { voltage-gated channel, shaker-related } \\
\text { subfamily, } \beta \text { - member I }\end{array}$ \\
\hline AKR6A5 & $\begin{array}{l}\text { KCNAB 2; hKvbeta2; potassium } \\
\text { voltage-gated channel, shaker-related } \\
\text { subfamily, } \beta \text { - member } 2\end{array}$ \\
\hline AKR6A9 & $\begin{array}{l}\text { KCNAB 3; potassium voltage-gated } \\
\text { channel, shaker-related subfamily, } \beta \text { - } \\
\text { member } 3\end{array}$ \\
\hline AKR7A2 & Aflatoxin aldehyde reductase; AFARI \\
\hline AKR7A3 & Aflatoxin aldehyde reductase; AFAR2 \\
\hline
\end{tabular}

and AKR7 family members show evidence of forming multimers, although it is unclear whether quaternary structure is essential for enzyme activity. $^{7-9}$ The single amino acid chain of, on average, 320 residues folds into a $(\alpha / \beta)_{8}$-barrel, an evolutionarily highly conserved structure originally observed in triose-phosphate isomerase (TIM) and therefore also known as a TIM barrel structure. In contrast to another 32 superfamilies adopting this motif, AKRs do not require metal ions for functionality, and bind $\mathrm{NAD}(\mathrm{P}) \mathrm{H}$ cofactor in an extended anti-conformation so that they can catalyse 4-pro- $R$ hydride transfer and thus belong to the large group of A-face oxidoreductases.

The spectrum of AKR substrates is wide and comprises aldehydes, ketones, monosaccharides, ketosteroids, ketoprostaglandins, bile acid precursors, chemical carcinogens and their metabolites for example, aflatoxin dialdehydes, nicotine-derived 4-(methylnitrosamino)-1-(3-pyridyl)-1-butanone and trans-dihydrodiols of polycyclic aromatic hydrocarbons. Substrate recognition and binding takes place at the C-terminal end of the ellipsoid $(\alpha / \beta)_{8}$-barrel body through interaction with amino acids within the three highly variable loops protruding out of the barrel structure. ${ }^{2,10,11}$ Indeed, sequence conservation among AKRs is lowest in these loops, compared with the $\alpha$-helices and $\beta$-strands forming the barrel structure itself, and suggests that AKRs evolved from a common ancestor by duplication and subsequent divergence to accommodate the binding of the structurally diverse substrates.

Duplication and diversification indeed seem to have been the driving force in the formation of vertebrate AKRs. AKR1B, AKR1C and AKR7A family members appear to be vertebrate specific and in the human genome their genes cluster on chromosomes 7,10 and 1 , respectively. ${ }^{5}$ Furthermore, the potential of these three subfamilies to give rise to new functional AKRs is reflected in the existence of several pseudogenes and putative new isoforms in the human genome. By contrast, the genes of all three human AKR6 family members reside in different chromosomal locations, show a high degree of conservation, even to plant AKR6 isoforms, and do not give rise to functional or non-functional (pseudogene) gene duplications.

\section{Biochemistry of AKRs}

Almost all AKRs catalyse the reduction of aldehydes and ketones to primary and secondary alcohols, respectively, by use of a reduced nicotinamide cofactor. The reactions involve an ordered bi-bi kinetic mechanism and general acid-base catalysis. $^{12-14}$ The cofactor binds first, followed by 
tethering of the substrate. In the reduction direction, the 4-pro- $R$ hydride from $\mathrm{NAD}(\mathrm{P}) \mathrm{H}$ is transferred to the substrate carbonyl group, followed by protonation of the carbonyl oxygen by a conserved tyrosine acting as a general acid. ${ }^{15-17}$ Oxidation proceeds in the reverse sequence with the tyrosine acting as a general base.

Asp 50, Tyr 55, Lys 84 and His 117 form the catalytic tetrad (based on residue numbering in AKR1C9), ${ }^{2,18,19}$ where the Tyr is the general acidbase. $^{17,20}$ Conservation of these residues is strong and underlines their role in catalysis. Asp 50 is present in 99 per cent, Tyr 55 in 97 per cent, Lys 84 in 97 per cent and His 117 in 88 per cent of all annotated AKRs on the AKR website. Consistent substitution of these key amino acids, however, especially of the histidine, is a characteristic for some AKR subfamilies. In AKR1D isoforms, which catalyse the $5 \beta$-reduction of $\Delta^{4}$-3-ketosteroids, glutamate replaces His $117 .^{2,21}$ Interestingly, mutational analysis in AKR1C9 demonstrates that this single amino acid substitution is sufficient to convert this 3-ketosteroid reductase to a steroid $5 \beta$ doublebond reductase. ${ }^{22,23}$ The reverse change of function has been hypothesised for the respective $(\mathrm{E} 120 \mathrm{H})$ mutation in AKR1D1. ${ }^{24}$ In all AKR6A isoforms, the conserved histidine is changed to asparagine. Based on crystal structure comparisons, Di Costanzo et al. hypothesised that in AKR6A isoforms the amino acid in this position is involved in substrate discrimination. ${ }^{25}$ AKR6A isoforms, better known as voltage-gated potassium channel $\beta$-subunits $(\mathrm{Kv} \beta$ or $\mathrm{KCNAB}$ ), accept a wide range of substrates, but their catalytic efficiency is significantly lower than other AKRs. ${ }^{26,27}$ It has been suggested that they may primarily function as redox state sensors in the regulation of potassium channel activity. ${ }^{27-29}$ The replacement of histidine with asparagine may provide more space to allow for the optimal positioning of bulky lipid and phospholipid aldehydes, formed as products of lipid peroxidation and oxidative stress.

Although these four conserved amino acids seem to play a crucial role in the catalytic mechanism, targeted mutation studies (eg in AKR $1 B 1^{15}$ and AKR $1 C 9^{30}$ ) did not indicate a complete loss in enzymatic activity and suggested that, in some instances, proximity of the hydride donor to the acceptor is sufficient to catalyse a reaction. Furthermore, some AKRs, such as $\rho$-crystallins in amphibian lenses (AKR1C10), appear to have been employed in this tissue basically for structural reasons, having lost almost all enzymatic activity despite unaltered characteristics of the cofactor and substrate binding site. ${ }^{31}$

\section{Nomenclature}

The nomenclature for the AKR superfamily, introduced and accepted by the Hugo Genome Nomenclature Committee (HGNC) in 1997, builds solely on sequence and not on functional similarity. Furthermore, systematic annotation through the AKR website requires proof of functionality of the protein in question. This evidence may be RNA or enzyme based, and aims to avoid the annotation of non-functional pseudogenes or predicted genes of unknown physiological function. Sequence submission for the assignment of a systematic AKR superfamily name should be sent directly to the AKR website (http://www.med. upenn.edu/akr/). ${ }^{3,4}$ The basic principle in naming a new AKR follows that for the cytochrome $\mathrm{P}$ (CYP) superfamily. ${ }^{32,33}$ All members of the superfamily are annotated with the suffix AKR. The 15 families (Figure 1) into which annotated AKRs fall have less than 40 per cent amino acid identity with each other. Subfamilies, denoted by a capital roman letter, share up to 60 per cent sequence identity. Individual members in the subfamily are numbered according to the chronology of sequence submission to the AKR website. Thus, AKR1A1 (human aldehyde reductase) belongs to family 1 , subfamily $A$ and is the first unique protein in the subfamily. If two sequences share 97 per cent or more sequence identity, they are considered as alleles and marked by an additional small roman letter (eg AKR1C10a and AKR1C10b). Two sequences sharing this high degree of identity are not regarded as alleles if they reside in different genomic locations, have distinct $3^{\prime}$ untranslated regions or differ in their enzymatic activities. It was also recommended that the corresponding gene be 


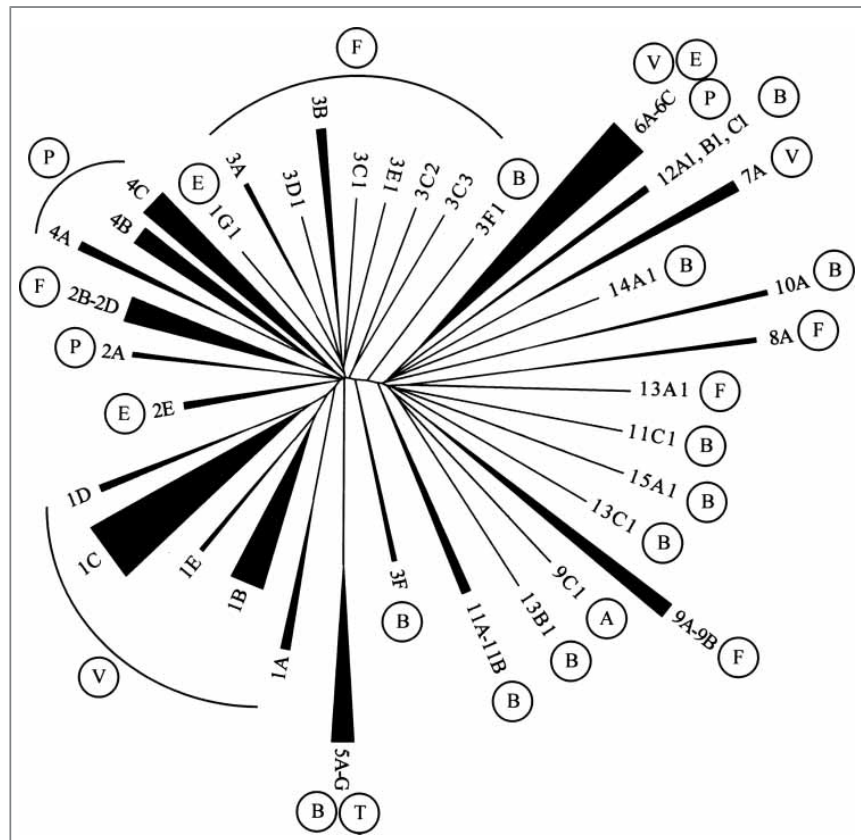

Figure I. Dendogram of all systemically annotated AKR enzymes. All sub-trees comprising more than a single member have been collapsed and the width of the resulting wedge reflects the number of annotated sequences in this category. Circled capital letters denote the species of origin. A, archeae; $\mathrm{B}$, bacteria; E, ecdysozoa; F, fungi; P, plants; T, trypanosomatidae; $\mathrm{V}$, vertebrate.

given the same name as the protein, but written in italics.

The nomenclature has the advantage that it assigns a unique gene name to each single AKR through establishing its amino acid sequence similarity to other families and subfamilies. In this way, it is independent of enzyme properties, species of origin and functional homologies. Therefore, it is not surprising that most families at the same time constitute clusters of closely related organisms rather than similar function, and that an AKR of a more distantly related organism will fall into a different subfamily (Figure 1). For example, bacterial AKRs form their own families with multiple subfamilies owing to the high diversification that has occurred in the 3,900 million years since bacteria and archeae split. At the same time, vertebrate, but especially mammalian, AKR sequences (mammalians appeared about 300 million years ago) form sequence clusters with multiple, highly similar members in each subfamily. Inspection of the vertebrate AKR sequences reveals that the AKR1 family evolved separately from families AKR6 and AKR7. Furthermore, only potassium channel $\beta$-subunit sequences have been so well conserved during evolution that plant and arthropod sequences both cluster in the AKR6 family.

\section{A search for AKRs on NCBI, HGNC and Ensembl}

Although the new nomenclature came into effect nearly 12 years ago — and has been widely accepted by the AKR research community - it has not yet become widely adopted by database annotators. This is especially true for non-mammalian AKRs. Of the 160 proteins annotated on the AKR website (http://www.med.upenn.edu/akr/), 62 give problematic results if searched with their systematic name against the National Center for Biotechnology Information (NCBI) database. For 45 AKRs, not a single database entry can be found. This illustrates that regular updates of protein and nucleotide annotations have failed to notice and acknowledge the proposed systematic nomenclature.

Annotation and nomenclature of human AKRs, however, are far more consistent. A database search for human AKRs at the National Institutes of Health (http://www.ncbi.nlm.nih.gov), the European Molecular Biology Laboratory (www. ensembl.org), the Sanger Institute (http://vega. sanger.ac.uk/index.html) and the HGNC (http:// www.genenames.org) identified 13 of the 14 proteins listed on the AKR website (Table 1). AKR1E2, which appears in all four databases under its synonym AKR1CL2, was originally annotated as LoopADR in 2000 and later named AKR1C like 2 (AKR1CL2), based on its sequence similarity to AKR1C isoforms. In 2003 and 2004, two publications described the enzymatic activity of this protein towards 9,10-phenanthrenequinone, but not steroid hormones, and termed it human testisspecific AKR (htAKR), based on its restricted expression pattern. ${ }^{34,35}$ The protein shares 74 per cent sequence identity with the previously described 
murine AKR $1 E 1^{36}$ and hence was systematically named AKR1E2.

\section{Unclassified and predicted human AKR database entries}

The database search for human AKRs at NCBI also revealed a number of other sequences annotated in context with AKRs. The total of 58 database entries comprised the 14 systematically annotated and well-described human AKRs and 12 separate annotations of pseudogenes. The search also revealed that in 22 cases, the respective protein belonged to a different protein family but appears in a biological context with an AKR. The remaining ten database entries (Table 2) resemble unclassified or predicted human AKR sequences that are related to three different AKR subfamilies: AKR1C, AKR1B and AKR7A.

$A K R 1 C L 1$ is located between $A K R 1 C 3$ and $A K R 1 C 4$ on chromosome 10. Ambiguous reports at NCBI describe two different forms of the gene. In one case, exons 1-4 of eight exons encode a 129-amino acid peptide (Accession No. NP001007537.1). This protein includes the complete catalytic tetrad but appears to be too short to be a functional AKR. In the second case, AKR1CL1, similarly to the other four AKR1C members, consists of nine exons and encodes a complete 326-amino acid protein (Accession No. EAW86451) with 68 per cent sequence identity to AKR1C1-4. The shorter version exactly matches

Table 2. Non-classified human AKRs in the NCBI database

\begin{tabular}{|c|c|c|c|c|c|}
\hline Name & $\begin{array}{l}\text { Alternative } \\
\text { names }\end{array}$ & Gene ID ${ }^{a}$ & $\begin{array}{l}\text { Chromosomal } \\
\text { location }\end{array}$ & Related to & Probable identity \\
\hline$A K R / B I I$ & $A R L, H S I$ & 9405 & 7 & AKRIB & Variance of $A K R / B / O$ \\
\hline$A K R / B / O L$ & tcag7.1260 & 441282 & $7 q 33$ & AKRIB & $\begin{array}{l}\text { New } A K R \text { or pseudogene } \\
\text { of } A K R I B / 0\end{array}$ \\
\hline LOC340888 & & 340888 & $10 q 21.3$ & AKRIB & $\begin{array}{l}\text { Retro-transposed } \\
A K R / B / 0 \text { pseudogene }\end{array}$ \\
\hline LOC643582 & & 643582 & $18 q 22.1$ & AKRIB & $\begin{array}{l}\text { Retro-transposed } \\
\text { AKRIBIO pseudogene }\end{array}$ \\
\hline$A K R / C L I$ & RAKc & 340811 & 10pI5.I & AKRIC & $\begin{array}{l}\text { New AKRIC or } \\
\text { pseudogene }\end{array}$ \\
\hline$A K R /$ C-pseudo & & 266745 & $10 p|5-10 p| 4$ & AKRIC & Variance of $A K R / C 2$ \\
\hline LOC6485I 7 & & 648517 & Unclear & AKRIC & $\begin{array}{l}\text { AKRIC pseudogene or } \\
\text { transcript variant }\end{array}$ \\
\hline LOCI00I34257 & & 100134257 & Unclear & AKRIC & $\begin{array}{l}\text { AKRIC pseudogene or } \\
\text { transcript variant }\end{array}$ \\
\hline LOC643789 & & 643789 & 10p|5.I & AKRIC & Pseudogene \\
\hline LOC648947 & & 648947 & 10p|5.I & AKRIC & $A K R I C L I$ pseudogene \\
\hline$t A K R$ & & 389932 & 10pI5.I & AKRIC & $A K R / C$ pseudogene \\
\hline$A K R 7 L$ & $\begin{array}{l}\text { AKR7A4, } \\
\text { AFAR3 }\end{array}$ & 246181 & Ip35-Ip36.I & AKR7A & $\begin{array}{l}\text { New AKR7A or } \\
\text { pseudogene }\end{array}$ \\
\hline AFARPI & & 246182 & $|p| 2$ & AKR7A & $\begin{array}{l}\text { Retro-transposed AKR7 } \\
\text { pseudogene }\end{array}$ \\
\hline
\end{tabular}

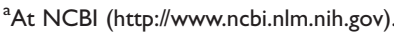


residues 1-129 of the longer form and resembles a differently transcribed and processed version of the same gene. A few messenger RNA (mRNA) sequences are annotated that match $A K R 1 C L 1$ with deletions and frameshifts, but a Blast search with the longer version against the expressed sequence tag (EST) database does not yield any AKR1CL1 transcripts. Therefore, it remains unclear whether AKR1CL1 is a processed pseudogene or a new functional AKR1C member.

$A K R 1 C$-pseudo is an old, discontinued entry that resembles $A K R 1 C 2$. AKR1C2 shares 97 per cent sequence identity with AKR1C1 but exhibits a unique expression pattern and substrate specificity. $^{37-41}$ LOC648517 and LOC100134257 contain sequence elements that completely match exons of AKR1C1 and AKR1C2. Their chromosomal location could not be determined, however, and therefore it is not clear whether they might be transcript variants of either $A K R 1 C 1$ or $A K R 1 C 2$, or are separate pseudogenes.

Four entries are related to AKR1B10. While $A K R 1 B 11$ is a discontinued record replaced by AKR1B10, LOC340888 and LOC643582 are Gnomon predictions and appear to be retrotransposed pseudogenes of $A K R 1 B 10$. AKR1B10L resides next to $A K R 1 B 10$ on chromosome 7 and also consists of ten exons. The two proteins share 91 per cent amino acid identity. The presence of a single EST from placenta (BX350113.2) that completely matches residues 23-209 of the predicted 316-amino acid AKR1B10L transcript suggests that AKR1B10L could indeed resemble a new and thus far uncharacterised human AKR family member. Alternatively, the presence of the mRNA could result from a processed pseudogene.

Two additional database entries relate to the AKR7 family. $A K R 7 L$ clusters together with $A K R 7 A 2$ and $A K R 7 A 3$ on chromosome 1p35-1p36.1., ${ }^{3,42}$ Alternative transcription leads to two different transcript variants. Variant 1 is the longer and comprises seven exons that encode a 331-amino acid protein. This form may indeed resemble a new functional AKR that has 92 per cent and 88 per cent identity with AKR7A3 and AKR7A2, respectively. Variant 2 lacks exons 4 and 5 and codes for a probably non-functional alternative peptide. This deletion results in a frameshifteliminating sequence similarity to AKRs in the last 80 amino acids of the 250-amino acid peptide. The AKR7 family pseudogene (AFARP1) is a retrotransposed $A K R 7$, where the complete processed $m R N A$ has reintegrated into the genome.

In addition, Barski et al. ${ }^{5}$ report on three putative AKRs not identified in the above-described database search. The database entry LOC643789 was originally designated as the $A K R 1 C$ pseudogene but was discontinued in March 2008. LOC648947 is a predicted gene with nine exons, encodes a 316-amino acid protein and shares 82 per cent sequence identity with the longer version of AKR1CL1; however, this protein sequence harbours a stop codon following amino acid 199. At least three mRNA sequences for this gene are present in the EST database. They do not completely match the predicted protein sequence of LOC648947, and a region corresponding to amino acids $192-227$ is deleted. As this deletion covers a region conserved in all AKR1C isoforms, it is very likely that LOC648947 represents a processed pseudogene of AKR1CL1. Finally, tAKR shows similarity to other AKR1C isoforms and the gene is located between $A K R 1 C L 2$ and $A K R 1 C 1$ on chromosome 10. Original annotation predicts a gene of 11 exons, with exons 3-11 encoding a putative protein with more than 300 amino acids. A transcript exists only for the first 125 amino acids, however, and hence $t A K R$ is thus far classified as a processed pseudogene.

In summary, systematic integration of these AKR database entries into the AKR nomenclature is still pending owing to a lack of sufficient experimental data to support an assignment.

\section{Annotated variants of AKRs}

Efforts have been made systematically to describe genetic variants and splice isoforms in annotated human $A K R s$ and to identify the location and classification of putative pseudogenes (Table 3). Data on single nucleotide polymorphisms (SNPs) are accumulating rapidly, and the SNP database 
Table 3. Genomic and transcriptomic variations in annotated human AKRs

\begin{tabular}{|c|c|c|c|c|c|c|c|}
\hline AKR & $\begin{array}{l}\text { Gene } \\
\text { ID }^{\mathbf{a}}\end{array}$ & $\begin{array}{l}\text { Chromosomal } \\
\text { location }\end{array}$ & $\begin{array}{l}\text { No. of SNPs }{ }^{\text {b }} \text { (in gene / } \\
\text { coding region/ } \\
\text { non-synonymous / } \\
\text { frameshift) }\end{array}$ & $\begin{array}{l}\text { SNP frequency (per } \\
\text { I kilobase: in genel } \\
\text { in coding region) }\end{array}$ & $\begin{array}{l}\text { Peptide } \\
\text { variants }\end{array}$ & $\begin{array}{l}\text { Additional } \\
\text { processed } \\
\text { transcripts }\end{array}$ & $\begin{array}{l}\text { Pseudogenes } \\
\text { on vega } \\
\text { (HGNC) }\end{array}$ \\
\hline$|A|$ & 10327 & $1 p 33-p 32$ & $154 / 9 / 5 / 1$ & $8.01 / 9.2$ & 3 & 10 & 0 \\
\hline$|B|$ & 231 & $7 q 35$ & $160 / 13 / 9 / 0$ & $9.53 / 13.67$ & 3 & 12 & $5(8)$ \\
\hline $\mathrm{ICI}$ & 1645 & |0p|5-p|4 & $160 / 16 / 6 / 0$ & $10.88 / 16.46$ & 3 & 2 & 0 \\
\hline IC2 & 1646 & $|0 p| 5-p \mid 4$ & $353 / 16 / 8 / 0$ & $12.5 / 16.46$ & I & I & 0 \\
\hline $1 C 3$ & 8644 & I0p|5-p|4 & $261 / 23 / 13 / 2$ & $19.61 / 23.66$ & I & 3 & 0 \\
\hline IE2 & 83592 & $10 p \mid 5.1$ & $211 / 12 / 7 / 0$ & $9.66 / 12.46$ & 2 & 4 & 0 \\
\hline $6 A 3$ & 7881 & $3 q 26.1$ & $1888 / 1 / 0 / 0$ & $4.51 / 0.81$ & 10 & 0 & 0 \\
\hline $6 A 5$ & 8514 & Ip36.3 & $4 / 4 / 8 / 2 / 1$ & $7.15 / 7.25$ & 8 & 6 & 0 \\
\hline 6 A9 & 9196 & $|7 p| 3 . \mid$ & $42 / 3 / 1 / 2$ & $6.24 / 2.47$ & I & 2 & 0 \\
\hline $7 A 2$ & 8574 & Ip36.13 & $71 / 10 / 8 / 0$ & $8.68 / 9.26$ & I & 0 & 4 \\
\hline $7 A 3$ & 22977 & $1 p 36.13$ & $67 / 12 / 5 / 0$ & $10.76 / 12.05$ & I & 0 & 0 \\
\hline
\end{tabular}

${ }^{a} A t \mathrm{NCBI}$ (http://www.ncbi.nlm.nih.gov).

beduced from the vega database (http://vega.sanger.ac.uk/index.html).

The table does not include the putative transcript variants and pseudogenes listed in Table 2.

build 130 (NCBI), as of 30th April 2009, contains over 17.8 million SNP clusters, with over 7.3 million SNPs located in genes. Nomenclature for SNPs in AKRs is based on that for sulfotransferase (SULT) genes ${ }^{43}$ and takes only coding SNPs into account. $^{4}$ Systematic annotation of the SNP is marked by the gene name followed by an asterisk, an Arabic number indicating synonymous (' 1 ') or non-synonymous ('2'-'n') character and a letter distinguishing between suballeles; the nomenclature is chronologically based. While this seems to be a feasible approach for synonymous and nonsynonymous SNPs, it neglects SNPs leading to frameshifts and those in the non-coding gene region that have the potential to alter the transcription efficiency, splicing and stability of mRNA. For all AKRs SNP frequency is significantly higher than the estimated $1 \mathrm{SNP} /$ kilobase. $^{44,45}$ Although the total number of SNPs per gene is only a snapshot in this time of extremely rapid data growth, it appears that some AKRs (eg AKR1D1, AKR6A3 and $A K R 6 A 9$ ) have accumulated significantly fewer single nucleotide variations in their coding region than others.

In addition to SNPs, other variations have been annotated over the years. The most well known are pseudogenes, and one or several have been identified for a few $A K R s$. For example, the pseudogene forms of $A K R 1 B 1$ are well documented and systematically annotated as $A K R 1 B 1 P 1-8$, where $\mathrm{P}$ stands for pseudogene. For example, $A K R 1 B 1 P 4$ denotes the fourth pseudogene annotated for $A K R 1 B 1$. Analysis of the non-systematically named AKRs (Table 2) suggests that many more pseudogenes, in addition to the few already annotated, might exist. This may also be the case for alternative splice forms, where the vega database (http://vega.sanger.ac.uk/index.html) from the Sanger Institute offers the most systematic 
annotation approach. Gene transcripts are numbered in the order of their annotation and designated with the suffix ' $-n$ ' added to the specific gene name. For example, $A K R 1 C 1$ has five different transcripts, annotated as AKR1C1-001 to AKR1C1-005. For half of the human $A K R s$, transcript processing leads to one or more alternative peptides. Furthermore, non-coding RNAs have been identified for 11 of the 14 genes. While, in the past, these fragments have been ignored, being perceived as resulting from errors in transcription or splicing, increasing evidence suggests that they play important regulatory functions (eg in gene expression, imprinting, RNA stability, splicing and translation). ${ }^{46}$ Their role in AKR expression and function remains to be elucidated.

\section{Summary}

Proteins of the AKR superfamily are present in all phyla and are characterised by their common threedimensional structure and reaction mechanism. Fourteen human AKRs have so far been annotated with systematic gene names in accordance with a systematic nomenclature provided through the AKR website that is based on amino acid sequence similarity and proof of protein functionality. Additional non-systematic annotations of human AKRs are present in the NCBI database that may resemble new, uncharacterised proteins or pseudogenes of existing AKRs. Furthermore, the rapid and continuous increase in data on SNPs and transcript variants challenges a systematic annotation of these phenomena and adds to the complexity of defining the functionality of a given gene product. Despite these complexities, the AKR website provides the most comprehensive and up-to-date portal for tracking the development of AKR annotation and provides links to in-depth information on each single AKR superfamily member in other databases.

\section{Acknowledgments}

The writing of this paper was funded in part by $\mathrm{NIH}$ grants P30-ES013508, R01-DK47015, R01-CA90744 and R01-CA39504 awarded to T.M.P.

\section{References}

1. Jez, J.M., Flynn, T.G. and Penning, T.M. (1997), 'A nomenclature system for the aldo-keto reductase superfamily', Adv. Exp. Med. Biol. Vol. 414, pp. $579-600$.

2. Jez, J.M., Bennett, M.J., Schlegel, B.P., Lewis, M. and Penning, T.M. (1997), 'Comparative anatomy of the aldo-keto reductase superfamily', Biochem. J. Vol. 326, pp. 625-636.

3. Hyndman, D., Bauman, D.R., Heredia, V.V. and Penning, T.M. (2003), 'The aldo-keto reductase superfamily homepage', Chem. Biol. Interact. Vol. 143-144, pp. 621-631

4. Drury, J.E., Hyndman, D., Jin, Y. et al. (2007), 'The aldo-keto reductase superfamily homepage: 2006 update', In: Weiner, H., Maser, E., Lindhal, R. et al. (eds), Enzymology and Molecular Biology of Carbonyl Metabolism, Purdue Press, West Lafayette, IN, pp. 184-197.

5. Barski, O.A., Tipparaju, S.M. and Bhatnagar, A. (2008), 'The aldo-keto reductase superfamily and its role in drug metabolism and detoxification', Drug Metab. Rev. Vol. 40, pp. 553-624.

6. Jin, Y. and Penning, T.M. (2007), 'Aldo-keto reductases and bioactivation/ detoxication', Annu. Rev. Pharmacol. Toxicol. Vol. 47, pp. 263-292.

7. Kozma, E., Brown, E., Ellis, E.M. and Lapthorn, A.J. (2002), 'The crystal structure of rat liver AKR7A1. A dimeric member of the aldoketo reductase superfamily', J. Biol. Chem. Vol. 277, pp. 16285-16293.

8. Kavanagh, K.L., Klimacek, M., Nidetzky, B. and Wilson, D.K. (2002), 'The structure of apo and holo forms of xylose reductase, a dimeric aldoketo reductase from Candida tenuis', Biochemistry Vol. 41, pp. 8785-8795.

9. Gulbis, J.M., Mann, S. and Mackinnon, R. (1999), 'Structure of a voltage-dependent $\mathrm{K}^{+}$channel $\beta$ subunit', Cell Vol. 97, pp. 943-952.

10. Bennett, M.J., Albert, R.H., Jez, J.M., Ma, H. et al. (1997), 'Steroid recognition and regulation of hormone action: Crystal structure of testosterone and $\mathrm{NADP}^{+}$bound to $3 \alpha$-hydroxysteroid/dihydrodiol dehydrogenase', Structure Vol. 5, pp. 799-812.

11. Couture, J.F., De Jesus-Tran, K.P., Roy, A.M., Cantin, L. et al. (2005), 'Comparison of crystal structures of human type $33 \alpha$-hydroxysteroid dehydrogenase reveals an "induced-fit" mechanism and a conserved basic motif involved in the binding of androgen', Protein Sci. Vol. 14, pp. 1485-1497.

12. Askonas, L.J., Ricigliano, J.W. and Penning, T.M. (1991), 'The kinetic mechanism catalysed by homogeneous rat liver $3 \alpha$-hydroxysteroid dehydrogenase. Evidence for binary and ternary dead-end complexes containing non-steroidal anti-inflammatory drugs', Biochem. J. Vol. 278, pp. 835-841.

13. Neuhauser, W., Haltrich, D., Kulbe, K.D. and Nidetzky, B. (1997), 'NAD(P)H-dependent aldose reductase from the xylose-assimilating yeast Candida tenuis. Isolation, characterization and biochemical properties of the enzyme', Biochem. J. Vol. 326, pp. 683-692.

14. Trauger, J.W., Jiang, A., Stearns, B.A. and Lograsso, P.V. (2002), 'Kinetics of allopregnanolone formation catalyzed by human $3 \alpha$-hydroxysteroid dehydrogenase type III (AKR1C2)', Biochemistry Vol. 41, pp. 13451-13459.

15. Bohren, K.M., Grimshaw, C.E., Lai, C.J., Harrison, D.H. et al. (1994), 'Tyrosine-48 is the proton donor and histidine-110 directs substrate stereochemical selectivity in the reduction reaction of human aldose reductase: Enzyme kinetics and crystal structure of the $\mathrm{Y} 48 \mathrm{H}$ mutant enzyme', Biochemistry, Vol. 33, pp. 2021-2032.

16. Penning, T.M. (1999), 'Molecular determinants of steroid recognition and catalysis in aldo-keto reductases. Lessons from $3 \alpha$-hydroxysteroid dehydrogenase', J. Steroid Biochem. Mol. Biol. Vol. 69, pp. 211-225.

17. Schlegel, B.P., Jez, J.M. and Penning, T.M. (1998), 'Mutagenesis of $3 \alpha$-hydroxysteroid dehydrogenase reveals a "push-pull" mechanism for proton transfer in aldo-keto reductases', Biochemistry Vol. 37, pp. 3538-3548.

18. Jez, J.M., Flynn, T.G. and Penning, T.M. (1997), 'A new nomenclature for the aldo-keto reductase superfamily', Biochem. Pharmacol. Vol. 54, pp. 639-647.

19. Bennett, M.J., Schlegel, B.P., Jez, J.M., Penning, T.M. and Lewis, M. (1996), 'Structure of $3 \alpha$-hydroxysteroid/dihydrodiol dehydrogenase complexed with $\mathrm{NADP}^{+}$, Biochemistry Vol. 35, pp. 10702-10711. 
20. Grimshaw, C.E., Bohren, K.M., Lai, C.J. and Gabbay, K.H. (1995), 'Human aldose reductase: $\mathrm{pK}$ of tyrosine 48 reveals the preferred ionization state for catalysis and inhibition', Biochemistry Vol. 34, pp. 14374-14384.

21. Kondo, K.H., Kai, M.H., Setoguchi, Y., Eggertsen, G. et al. (1994), 'Cloning and expression of cDNA of human $\Delta^{4}$-3-oxosteroid $5 \beta$-reductase and substrate specificity of the expressed enzyme', Eur. J. Biochem. Vol. 219, pp. $357-363$.

22. Penning, T.M., Ma, H. and Jez, J.M. (2001), 'Engineering steroid hormone specificity into aldo-keto reductases', Chem. Biol. Interact. Vol. $130-132$, pp. 659-671.

23. Jez, J.M. and Penning, T.M. (1998), 'Engineering steroid $5 \beta$-reductase activity into rat liver $3 \alpha$-hydroxysteroid dehydrogenase', Biochemistry Vol. 37, pp. 9695-9703.

24. Drury, J.E. and Penning, T.M. (2007), ' $\Delta^{4}$-3-ketosteroid $5 \beta$-reductase (AKR1D1): Properties and role in bile acid synthesis', In: Weiner, H., Maser, E., Lindhal, R. et al. (eds), Enzymology and Molecular Biology of Carbonyl Metabolism, Purdue Press, West Lafayette, IN, pp. 332-340.

25. Di Costanzo, L., Penning, T.M. and Christianson, D.W. (2009), 'Aldo-keto reductases in which the conserved catalytic histidine is substituted', Chem. Biol. Interact. Vol. 178, pp. 127-133.

26. Tipparaju, S.M., Barski, O.A., Srivastava, S. and Bhatnagar, A. (2008), 'Catalytic mechanism and substrate specificity of the $\beta$-subunit of the voltage-gated potassium channel', Biochemistry Vol. 47, pp. 8840-8854.

27. Weng, J., Cao, Y., Moss, N. and Zhou, M. (2006), 'Modulation of voltage-dependent Shaker family potassium channels by an aldo-keto reductase', J. Biol. Chem., Vol. 281, pp. 15194-15200.

28. Tipparaju, S.M., Saxena, N., Liu, S.Q., Kumar, R. and Bhatnagar, A. (2005), 'Differential regulation of voltage-gated $\mathrm{K}^{+}$channels by oxidized and reduced pyridine nucleotide coenzymes', Am. J. Physiol. Cell Physiol. Vol. 288, pp. C366-C376.

29. Pan, Y., Weng, J., Cao, Y., Bhosle, R.C. and Zhou, M. (2008), 'Functional coupling between the Kv1.1 channel and aldoketoreductase Kvß1', J. Biol. Chem., Vol. 283, pp. 8634-8642.

30. Schlegel, B.P., Ratnam, K. and Penning, T.M. (1998), 'Retention of NADPH-linked quinone reductase activity in an aldo-keto reductase following mutation of the catalytic tyrosine', Biochemistry Vol. 37, pp. 11003-11011.

31. Fujii, Y., Watanabe, K., Hayashi, H., Urade, Y. et al. (1990), 'Purification and characterization of rho-crystallin from Japanese common bullfrog lens', J. Biol. Chem. Vol. 265, pp. 9914-9923.

32. Dayhoff, M.O., Barker, W.C. and Hunt, L.T. (1983), 'Establishing homologies in protein sequences', Methods Enzymol. Vol. 91, pp. 524-545.

33. Nebert, D.W., Adesnik, M., Coon, M.J. et al. (1987), 'The P450 gene superfamily: recommended nomenclature', DNAVol. 6, No. 1, pp. 1-11.
34. Nishinaka, T., Azuma, Y., Ushijima, S., Miki, S. et al. (2003), 'Human testis specific protein: A new member of aldo-keto reductase superfamily', Chem. Biol. Interact. Vol. 143-144, pp. 299-305.

35. Azuma, Y., Nishinaka, T., Ushijima, S., Soh, J. et al. (2004), 'Characterization of htAKR, a novel gene product in the aldo-keto reductase family specifically expressed in human testis', Mol. Hum. Reprod. Vol. 10, pp. $527-533$.

36. Bohren, K.M., Barski, O.A. and Gabbay, K.H. (1997), 'Characterization of a novel murine aldo-keto reductase', $A d v$. Exp. Med. Biol. Vol. 414, pp. $455-464$.

37. Ciaccio, P.J. and Tew, K.D. (1994), 'cDNA and deduced amino acid sequences of a human colon dihydrodiol dehydrogenase', Biochim. Biophys. Acta Vol. 1186, pp. 129-132.

38. Qin, K.N., New, M.I. and Cheng, K.C. (1993), 'Molecular cloning of multiple cDNAs encoding human enzymes structurally related to $3 \alpha$-hydroxysteroid dehydrogenase', J. Steroid Biochem. Mol. Biol. Vol. 46, pp. 673-679.

39. Stolz, A., Hammond, L., Lou, H., Takikawa, H. et al. (1993), 'cDNA cloning and expression of the human hepatic bile acid-binding protein. A member of the monomeric reductase gene family', J. Biol. Chem. Vol. 268, pp. $10448-10457$.

40. Penning, T.M., Jin, Y., Heredia, V.V. and Lewis, M. (2003), 'Structure-function relationships in $3 \alpha$-hydroxysteroid dehydrogenases: A comparison of the rat and human isoforms', J. Steroid Biochem. Mol. Biol. Vol. 85, pp. 247-255.

41. Penning, T.M., Jin, Y., Steckelbroeck, S., Lanisnik-Rizner, T. and Lewis, M. (2004), 'Structure-function of human $3 \alpha$-hydroxysteroid dehydrogenases: Genes and proteins', Mol. Cell Endocrinol. Vol. 215, pp. 63-72.

42. Praml, C., Savelyeva, L. and Schwab, M. (2003), 'Aflatoxin B1 aldehyde reductase (AFAR) genes cluster at 1p35-1p36.1 in a region frequently altered in human tumour cells', Oncogene Vol. 22, pp. 4765-4773.

43. Blanchard, R.L., Freimuth, R.R., Buck, J., Weinshilbourn, R.M. and Coughtrie, M.W. (2004), 'A proposed nomenclature system for the cytosolic sulfotransferase (SULT) superfamily', Pharmacogenetics Vol. 14, pp. 199-211.

44. Kwok, P.Y., Deng, Q., Zakeri, H., Taylor, S.L. and Nickerson, D.A. (1996), 'Increasing the information content of STS-based genome maps: Identifying polymorphisms in mapped STSs', Genomics Vol. 31, pp. 123-126.

45. Wang, D.G., Fan, J.B., Siao, C.J., Berno, A. et al. (1998), 'Large-scale identification, mapping, and genotyping of single-nucleotide polymorphisms in the human genome', Science Vol. 280, pp. 1077-1082.

46. Mattick, J.S. and Makunin, I.V. (2006), 'Non-coding RNA', Hum. Mol. Genet. Vol. 15, pp. R17-R29. 